\title{
Formal Alternatives as a Solution to the Proviso Problem
}

\author{
Raj Singh \\ Massachusetts Institute of Technology
}

\section{Introduction}

This paper investigates a particular problem concerning presupposition accommodation. Let $\psi$ and $X$ be sentences, and suppose $\psi$ presupposes $X$. We represent this as $\psi\{X\}{ }^{1}$ Suppose the speaker and hearer of a conversation are in context $c$. Suppose further that the speaker wishes to update the context $c$ with the information in $\psi$, an operation we represent as $c[\psi\{X\}] . \psi\{X\}$ imposes a requirement on $c$ that has to be met for the update to take place: $c$ already has to entail $X$. In terms of update operations, this means that if we try updating $c$ with $X$, we just get back $c$ : $c[X]=c$. When this condition is met, we say that $c$ satisfies $X$, or that $c$ is a fixed point for $[X]$. But what if $c$ is not a fixed point for $[X]$ ? What happens when the condition is not met?

There are at least two possible outcomes: (i) either communication breaks down, or (ii) we make some repair $[Y]$ to $c$ so that update will be defined after all, i.e. we update $c$ with $Y, c[Y]$, so that $c[Y]$ ends up satisfying $X$. Call this repair process accommodation Lewis (1979). We then update the new context resulting from accommodation of $Y$ with $\psi, c[Y][\psi]$. The question I explore in these pages is: Is there anything more to say about this repair process than simply "do something?" By detailed investigation of a particular puzzle in the theory of presupposition accommodation, this paper will: (i) Present empirical evidence that there are formal restrictions on the allowable repairs $[Y]$ that can be made, and (ii) Derive the restricted set of allowable repairs from the instructions encoded in the contextchange potentials defined in Heim (1983). We will see that the derivation of the set of alternatives makes crucial reference to the formal descriptions of context-change potentials (context-change potential descriptions, or CCPDs). Thus, the system

For much helpful discussion on the thoughts presented here, I would like to thank Pranav Anand, Asaf Bachrach, Chris Barker, Hiba Bou Akar, Greg Carlson, Emmanuel Chemla, Noam Chomsky, Mike Collins, Cleo Condoravdi, Veneeta Dayal, Paul Egré, Ted Gibson, Noah Goodman, Aravind Joshi, Lauri Karttunen, Ali Mohammad, Jeff Pelletier, Orin Percus, Alejando Pérez Carballo, Stanley Peters, Chris Potts, Philippe Schlenker, Florian Schwartz, Ken Shan, Benjamin Spector, Luke Zettlemoyer, two anonymous SALT reviewers, and especially Roni Katzir. I was also glad of thoughtful comments and questions from audiences at MIT's LF Reading Group, XeroxParc, the MIT-Paris Workshop on Implicatures and Presupposition at the ENS in Paris, and SALT 17 at the University of Connecticut in Storrs, CT. I am most indebted to: Kai von Fintel, Danny Fox, Irene Heim, and Bob Stalnaker, for their very valuable and dedicated guidance. In a different sort of way, I should thank the Social Sciences and Humanities Research Council of Canada for their support (Number 752-2004-0542), and the MIT-France Seed Fund for Collaborative Research on Presupposition and Implicatures.

${ }^{1}$ We follow here as closely as possible the notation of Geurts (1996). 
here agrees with Geurts (1996) that a more syntactic approach to presupposition accommodation seems called for.

\section{The Proviso Problem}

Consider sentences of the form $\ulcorner$ if $\phi$, then $\psi\{X\}\urcorner$. "Satisfaction theories" of presupposition (Karttunen 1974, Stalnaker 1974, Heim 1983, Beaver 2001, von Fintel 2006, among others) predict that the semantic presupposition of this sentence is $\ulcorner$ if $\phi$, then $X\urcorner .^{2}$ A rather straightforward proposal concerning accommodation would be the following: when $c$ does not satisfy the presupposition of $\ulcorner$ if $\phi$, then $\psi\{X\}$, i.e. when $c$ is not a fixed point for [if $\phi$, then $X$ ], we repair $c$ by updating it with the semantic presupposition, and then we update the result with the content of the sentence: $c$ [if $\phi$, then $X$ ] [if $\phi$, then $\psi$ ]. However, Gazdar (1979), van der Sandt (1992) and Geurts $(1996,1999)$ observe that oftentimes the repair we make is not [if $\phi$, then $X]$, but rather $[X]$ simpliciter:

(1) If John flies to Toronto, his sister will pick him up from the airport

Semantic Presupposition If John flies to Toronto, he has a sister

Actual Accommodation John has a sister

This fact teaches us that we need to supplement satisfaction theories of presupposition with some further theoretical construct, call it $P$, which, upon being fed a conditional presupposition $\ulcorner$ if $\phi$, then $\mathrm{X}\urcorner$ by the grammar, returns the stronger alternative $X$ as the repair that should be made. However, note that conditional presuppositions are not always strengthened in this fashion:

(2) If John is a scuba diver, he'll bring his wetsuit ${ }^{3}$

Semantic Presupposition If John is a scuba diver, he has a wetsuit

Actual Accommodation If John is a scuba diver, he has a wetsuit

Thus, the output of $P$ is not deterministic: when the grammar inputs a conditional presupposition, $P$ will sometimes output the grammar's input, and will sometimes output something stronger. To complicate matters further, Geurts (1996, 1999) notes that when conditional presuppositions $\ulcorner$ if $\phi$, then $X\urcorner$ are triggered under factive verbs like know, they are never strengthened to $X$ :

(3) Mary knows that if John flies to Toronto, he has a sister

Semantic Presupposition If John flies to Toronto, he has a sister

Actual Accommodation If John flies to Toronto, he has a sister

\footnotetext{
${ }^{2}$ Other theories that make this prediction include Karttunen and Peters (1979), van Rooij (2007), and Schlenker (2007).

${ }^{3}$ From Geurts (1996).
} 
To summarize:

(4) If $\phi$, then $\psi\{X\}$

Semantic Presupposition If $\phi$, then $X$

Accommodation Possibilities $\{$ If $\phi$ then $X, X\}$

(5) $\quad K_{m}($ If $\phi$, then $X)$

Semantic Presupposition If $\phi$, then $X$

Accommodation Possibilities $\{$ If $\phi$ then $X\}$

For our purposes, we may state the proviso problem as follows:

The Proviso Problem When the semantics generates conditional presuppositions $\ulcorner$ if $\phi$, then $\mathrm{X}\urcorner$, is there a characterization of $P$ that can predict when conditional presuppositions can and cannot be strengthened and, when they can, when they will and when they will not be strengthened and, when they will be strengthened, what exactly they will be strengthened to?

I aim in this paper to provide an explicit characterization of $P$.

\section{An Existing Theory of $P$ : Accommodation as Holistic Inference}

I am not by any means the first to attempt to provide a solution to the proviso problem. Beaver (2001), von Fintel (2006), and Heim (2006) (henceforth BvFH) represent one line of response from the satisfaction theory camp. ${ }^{4}$ The response goes as follows. As a matter of presupposition projection, satisfaction theories correctly predict that conditional sentences $\ulcorner$ if $\phi$, then $\psi\{X\}\urcorner$ and knowledge attribution sentences $\left\ulcorner K_{m}\right.$ (If $\phi$, then $\left.\left.X\right)\right\urcorner$ semantically presuppose $\ulcorner$ if $\phi$, then $X\urcorner$. The semantic presupposition is the minimal amount of information that needs to be added to $c$ in order to ensure that update is defined. How does the minimal requirement, generated by the grammar, relate to the actual repair that's made in the course of communication? That's not a matter of concern for the grammar. It can only say: "I need such and such information to hold in the common ground." Common sense/plausibility reasoning will then be responsible for deciding what to accommodate.

In its most general characterization, such reasoning takes the form: (i) Generate a set of hypotheses $\mathcal{H}$ for accommodation, (ii) Select a member of $\mathcal{H}$ according to some criterion. This account needs to tell us two things. First, what is the space of hypotheses? Second, what is the relevant decision criterion? I follow here the treatment of Heim (2006), since it is fairly explicit concerning these two components.

\footnotetext{
${ }^{4}$ Karttunen and Peters (1979), Beaver (2006), Pérez Carballo (2007), and van Rooij (2007) represent other responses. Due to space limitations, I will not have occasion to discuss these approaches in any great detail here.
} 
Heim's proposal takes $\mathcal{H}$ to be a set of "salient propositions" containing at least the semantic presupposition. She leaves the concept of "salience" unanalyzed and stipulates the hypothesis space of salient propositions for $\ulcorner$ if $\phi$ then $\psi\{X\}\urcorner$ to be $\mathcal{H}=\{$ if $\phi$ then $X, X\}$. Given this hypothesis space, what is the relevant decision criterion?

$\mathrm{BvFH}$ and Beaver and Zeevat (To appear) suggest that the hearer performs some kind of plausibility reasoning in making her decision about what to accommodate. Crucially, this reasoning process does not compare the plausibility of the propositions in $\mathcal{H}$. For note that since $X$ asymmetrically entails $\ulcorner$ if $\phi$ then $X\urcorner$ there is no reasonable notion of plausibility according to which the former should come out as more plausible than the latter. As we've seen, however, $X$ is sometimes the preferred accommodation. von Fintel (2006) and Heim (2006) suggest that the plausibility comparison is not over $\mathcal{H}$, but rather over $\mathcal{H} *=\left\{\square_{S}(\right.$ if $\phi$ then $X) \wedge$ $\left.\neg \square_{S} X, \square_{S} X\right\}$, where by " $\square_{S} Z$ " we mean something like 'the speaker takes it for granted that $Z$ ' (Heim 2006) or 'the speaker intends it to be common ground that $Z$ ' (von Fintel 2006). ${ }^{5}$ Note that the members of $\mathcal{H} *$ do not stand in any entailment relation. The hearer now compares the plausibility of the members of $\mathcal{H} *$ and comes to a conclusion about what to accommodate based on this comparison.

Heim (2006) derives $\mathcal{H} *$ from $\mathcal{H}$ in two steps. First, $\mathcal{H}$ is tranformed into $\square_{S} \mathcal{H}=\left\{\square_{S} p: p \in \mathcal{H}\right\}$. Second, each member of $\square_{S} \mathcal{H}$ is exhaustified: $\mathcal{H} *=$ $\left\{\operatorname{exh}\left(\square_{S} \mathcal{H}\right)\left(\square_{S} p\right): \square_{S} p \in \square_{S} \mathcal{H}\right\}{ }^{6} \mathcal{H} *$ is then used to impose an ordering on the members of $\mathcal{H}$ : for all $r, s \in \mathcal{H}: r>s$ iff $\operatorname{exh}\left(\square_{S} \mathcal{H}\right)(r)$ is more plausible than $\operatorname{exh}\left(\square_{S} \mathcal{H}\right)(s)$. The decision criterion is then: select the maximal member of $\mathcal{H}$ with respect to the order $>$.

Let us work through some examples to get a sense for how this reasoning works. Begin with (1): If John flies to Toronto, his sister will pick him up from the airport. $\mathcal{H}=\{$ If John flies to Toronto he has a sister, John has a sister $\}$. The hearer then asks herself: which is more plausible, (i) that the speaker takes for granted that John has a sister on the condition that he flies to Toronto, but not unconditionally, or (ii) that the speaker takes for granted that John has a sister whether or not he flies to Toronto? Since (ii) is more plausible than (i), the accommodation is that John has a sister.

Let us turn now to example (2): If John is a scuba diver, he'll bring his wetsuit. Here, $\mathcal{H}=\{$ If John is a scuba diver he has a wetsuit, John has a wetsuit $\}$. The hearer again asks herself: which is more plausible, (i) that the speaker takes for granted that John has a wetsuit on the condition that he is a scuba diver, but not unconditionally, or (ii) that the speaker takes for granted that John has a wetsuit whether or not he's a scuba diver? In this case (i) is more plausible, so the

\footnotetext{
${ }^{5}$ I read Beaver (2001) and Beaver and Zeevat (To appear) as claiming that the plausibility reasoning is over hypotheses about the speaker's beliefs.

${ }^{6}$ Where $B$ is a set of propositions, $q$ a proposition, Heim gives the following semantics for exhaustivity: $\operatorname{exh}(B)(q)=q \wedge \forall u \in B(u \rightarrow(q \subset u))$. Kai von Fintel (p.c.) has raised the question of why we should be comparing exhaustified alternatives. I do not know the answer to this question. In von Fintel's system you only compare target common grounds, not exhaustified propositions generated from $\mathcal{H}$. The issue I raise below for this general line of thinking comes up either way.
} 
accommodated proposition is that John has a wetsuit if he's a scuba diver.

I believe this proposal is essentially correct. However, there is a non-trivial question that it leaves unanswered, viz. what constraints, if any, are imposed on hypothesis spaces $\mathcal{H}$ ? Recall that Heim's system is fully formalized but for the stipulation of the set of alternatives. The need to consider more than the semantic presupposition when deciding what to accommodate was established by (1). But we haven't any principle in place that can tell us, for any proposition $p$ that is logically stronger than the semantic presupposition, whether $p \in \mathcal{H}$ or $p \notin \mathcal{H}$.

The goals of my paper are: (i) to argue that an adequate theory of accommodation needs to solve this decision problem, and (ii) to provide an algorithm that does so. The argument proceeds in three steps. I begin by providing empirical evidence that not all logically possible strengthenings of the semantic presupposition are available for accommodation. Second, I use this evidence to argue that the restrictions on the hypothesis space must be formal in nature. I then provide a procedure that uses the CCPD of a sentence $S^{8}$ to generate its hypothesis space $\mathcal{H}$. Since the only information used by the procedure is the syntax of $S$ 's CCP, the set of alternatives is context-invariant. It is for this reason that I call the alternatives formal alternatives. This set of alternatives $\mathcal{H}$ can then be plugged into the BvFH accommodation system to make predictions about what will and will not get accommodated. As a consequence, the BvFH accommodation system will be entirely mechanized.

Before filling in the details, let me sketch the rough form of the solution I offer to the problem defined by the data in (1)-(3). For sentences of the form $\ulcorner$ if $\phi$, then $\psi\{X\}\urcorner$, my procedure will output $\mathcal{H}=\{$ if $\phi$ then $X, X\} .{ }^{9}$ For knowledgeattribution sentences $\left\ulcorner K_{m}\right.$ (If $\phi$, then $\left.\left.X\right)\right\urcorner$ the procedure will generate $\mathcal{H}=\{$ if $\phi$ then $X$ \}. It therefore follows from the procedure that the reason you don't find strengthening under know is that the strengthened alternative is just not generated, i.e. it's not really an alternative. ${ }^{10}$

\footnotetext{
${ }^{7}$ It is for this reason that I call such systems "holistic." See also Thomason et al. (2006) for a theory of accommodation couched within a framework of holistic, abductive, inference.

${ }^{8}$ Recall from the Introduction that by 'the CCPD of sentence $S$ ' I mean the description of $S$ 's CCP.

${ }^{9}$ These are to be construed as sentences now.

${ }^{10} \mathrm{My}$ proposal therefore stands in contrast with all the other proposals developed by satisfaction theorists in response to the proviso problem. The general view has been that it is principles of conversational/plausibility reasoning, not restrictions on hypothesis spaces, that are responsible for the variability in the strength of the accommodated proposition: Beaver (2006) argues that (1) and (3) come with different relevance presuppositions, Heim (2006) argues that they have different implicatures, Pérez Carballo (2007) and van Rooij (2007) argue that (3) makes the beliefs of the subject of the knowledge ascription relevant, which is enough to block default assumptions needed to generate strengthening.
} 


\section{A Revised Theory of $P$ : On the Modularity of Accommodation}

\subsection{The Need for a Modular Theory of Accommodation: Restricted Alternatives}

One learns a fair amount of information from a sentence. In general, we can visualize the flow of information generated by the utterance of sentence $\ulcorner\Psi\{X\}\urcorner$ in context $c$ as the following update sequence: $c[Y][\psi]\left[Z_{1}\right] \ldots\left[Z_{k}\right]$, where $Y$ is what has been accommodated, and the $Z_{i}$ are further inferences. ${ }^{11}$ Given the many inferences drawn from a sentence, how does one probe with any generality that part of the update corresponding to presupposition accommodation?

It turns out that we have in place a diagnostic, the so-called Hey Wait a Minute Test, that can serve as a test for distinguishing presupposition and assertion (von Fintel 2004b,c). I argue that the HWAMT can be used to help us characterize the hypothesis space for accommodation:

The Hey Wait a Minute Test In response to the speaker's utterance $\ulcorner\psi\{X\}\urcorner$ in a context $c$ not satisfying $X$, iff $Y$ is what is to be accommodated, $c[Y][\psi]$, the hearer may object Hey wait a minute! I didn't know Y!

Here are some examples of the HWAMT in action:

(6) S: John's sister will pick him up from the airport.

H: Hey wait a minute! I didn't know John has a sister!

(7) S: If John flies to Toronto, his sister will pick him up from the airport.

H: Hey wait a minute! I didn't know John has a sister!

This last example is especially important because it teaches us that the HWAMT probes not only the minimal repair, but rather whatever it is that is supposed to be accommodated.

We now employ the HWAMT to argue that plausibility reasoning over an unrestricted search space cannot be responsible for the accommodation patterns we find. ${ }^{12}$ Let's assume that accommodation $i s$ governed by plausibility reasoning over an unrestricted search space.

(8) S: Since John is president of Morgan Stanley, his car must be parked in the garage.

Minimal Repair $\mathrm{X}=\mathrm{John}$ has a car

\footnotetext{
${ }^{11}$ We adopt here the idea from Stalnaker (1998) and von Fintel (2006) that there are timing principles governing accommodation, in that it happens after the sentence has been uttered but before update with its content had had a chance to take place.

${ }^{12}$ I put aside here the idea that $\mathcal{H}$ is contrained by salience, since it is not clear to me what does and does not constitute a salient enough possibility to be considered for membership in $\mathcal{H}$. Other natural constraints on the search space come to my mind, eg. relevance. But then we run into the well-known frame problem (Fodor 1983). Until some account of relevance is offered that allows us to avoid the frame problem, I will put this consideration aside.
} 
Alternative Repairs $\{\mathrm{A}=\mathrm{John}$ has an expensive car, $\mathrm{B}=\mathrm{J}$ ohn has a cheap car,...\}

Let us assume, for simplicity of discussion, that $\mathcal{H}=\{X, A, B\}$. By Heim's procedure, $\square_{S} H=\left\{\square_{S} X, \square_{S} A, \square_{S} B\right\}$. Let us assume further that it is common ground that the speaker is John's best friend, and rides with him regularly (i.e. so that he is intimately acquainted with John and his car). ${ }^{13}$ Now consider $H *$, which we get by exhaustifying each member of $\square_{S} H$. One of the members of $H *$ is $\operatorname{exh}\left(\square_{S} \mathcal{H}\right)\left(\square_{S} X\right)=\square_{S} X \wedge \neg \square_{S} A \wedge \neg \square_{S} B$, which conveys the information that the speaker takes it for granted that the speaker has a car, but doesn't know whether the car is cheap or expensive. Assuming the speaker is well-informed about such matters, this contradicts information established in the common ground (viz. our assumption about the speaker's relation to John and his car), and so has zero probability. The remaining members of $H *$ are simply the propositions that the speaker takes it for granted that John's car is expensive and that the speaker takes it for granted that John's car is cheap. The plausibility of each, given our assumptions about the speaker's information, reduces to the plausibility of John's car being cheap or expensive. Of these, given what we know about salaries of CEOs of such companies, their preferences in vehicles, etc. the more plausible alternative is that John's car is expensive. As such, according to the BvFH account, accommodation of $A$ should be the optimal choice, i.e. the first of the following update sequences should be preferred: ${ }^{14}$

(9) Update Sequence 1: $c[A][S]$

(10) Update Sequence 2: $c[X][S]$

Under the HWAMT, contra BvFH's prediction, it seems only $X$ can go in for accommodation, not the more plausible $A$ :

(11) \#Hey wait a minute! I didn't know John has an expensive car!

(12) Hey wait a minute! I didn’t know John has a car!

The HWAMT indicates that what gets accommodated is not the more plausible information that John has an expensive car, but rather the information that John has a car, with no indication of the value of that car. Whatever the nature of the inference to John's car being expensive, the HWAMT teaches us that it has nothing to do with presupposition accommodation. More schematically, that inference would be one of the $Z_{i}$ in our update video $c[Y][\psi]\left[Z_{1}\right] \ldots\left[Z_{k}\right]$, and not $Y$.

Here is another example:

Context: John's family has a long history of breeding German Shepherds (and only German Shepherds). Consider it common ground that any member of the

\footnotetext{
${ }^{13}$ As far as I can tell, nothing crucial hinges on these assumptions.

${ }^{14}$ It is clearer that this should be so when reasoning about speaker belief than when reasoning about target common grounds as von Fintel (2006) has proposed.
} 
family need not have a dog, but if they do, it must be a German Shepherd. Thus, John's having a dog is contextually equivalent to his having a German Shepherd. John's friend, Bill, pays a surprise visit to his place. John's cousin, Mary, answers:

(13) Bill: Hi Mary. Is John home?

Mary: Um, no, he's out walking his dog.

Now consider the following update sequences:

(14) Update Sequence 1: c[John has a German Shepherd][John is out walking his $\operatorname{dog}$ ]

(15) Update Sequence 2: c[John has a dog][John is out walking his dog]

Note that in this case, since having a dog and having a German Shepherd are contextually equivalent, the target common grounds are identical. Indeed, if $\{\mathrm{John}$ has a dog, John has a German Shepherd $\} \subset \mathcal{H}$, the procedure from above predicts that (14) will be an acceptable update sequence if and only if (15) is. However, the HWAMT argues against this prediction:

(16) \#Hey wait a minute! I didn't know John has a German Shepherd!

(17) Hey wait a minute! I didn't know John has a dog!

This example teaches us even more strikingly than the previous one that what can go in for $Y$ is formally restricted. In this example, even though $c$ [John has a dog $]=c$ [John has a German Shepherd $]$, the HWAMT shows us that only the former update sequence is allowed. The accidental contextual equivalence seems to be irrelevant for the accommodation system. We take such facts to indicate that, contra $\mathrm{BvFH}$, presupposition accommodation is not governed by plausibility reasoning over an unrestricted search space. ${ }^{15}$ In the next section I will state some generalizations about the ways in which the hypothesis spaces for atomic and conditional sentences are restricted. I will expand the discussion to other sentences once I show (in Section 4.3) how these generalizations can be derived from CCPDs.

\subsection{Generalizations About Hypothesis Spaces for Accommodation: Atomic and Conditional Sentences}

The HWAMT above indicates that in atomic sentences the only allowable repair is the lexically encoded presupposition of the sentence itself:

Hypothesis Spaces 1 (Atomic Sentences) When updating $c[Y][\psi\{X\}]$, the hypothesis space for accommodation is $\mathcal{H}=\{X\}$, i.e. the only value $Y$ can take is $Y=X$.

\footnotetext{
${ }^{15}$ I should mention that several people (Noam Chomsky, Kai von Fintel, Irene Heim, Roni Katzir, and an anonymous SALT reviewer) have pointed out examples of felicitous uses of the HWAMT which do not seem to probe information represented by a formal alternative. I believe I have a response to the counter-examples, but space limitations prevent me from engaging in further discussion here.
} 
The case of conditionals $\left\ulcorner\right.$ if $\phi_{1}$ and $\ldots$ and $\phi_{k}$, then $\left.\psi\{X\}\right\urcorner$ is slightly more complicated, as we've already seen, for in conditionals there is variability in the allowable repairs. For instance, we've seen that when $k=1$, the hypothesis space seems to be: $\{$ If $\phi$ then $X, X\}$. This suggests the following generalization: The hypothesis space for accommodation in conditional sentences is composed of the semantic presupposition of the conditional plus the presupposition of its consequent. Unfortunately, the proposal doesn't quite work, as the following example due to Geurts (1996) shows:

(18) If John is a scuba diver and wants to impress his girlfriend, he'll bring his wetsuit

The hypothesis space according to this proposal would be: \{If John is a scuba diver and wants to impress his girlfriend he has a wetsuit, John has a wetsuit $\}$. However, as Geurts (1996) points out, we accommodate from (18) the information that if John is a scuba diver, he has a wetsuit. This corresponds to no element of the hypothesis space. ${ }^{16}$ It seems we need to get "inside" the conditional. In this case, we need at least If John is a scuba diver, he has a wetsuit to be an alternative. This suggests that in conditional sentences, the hypothesis space is composed of all Left-Strengthenings of the minimal repair:

Hypothesis Spaces 2 (Conditionals) When updating $c[Y]$ Iif $\phi_{1}$ and $\ldots$ and $\phi_{k}$, then $\psi\{X\}]$, the hypothesis space for accommodation is all Left-Strengthenings of the semantic presupposition: $\mathcal{H}=\left\{\right.$ if $\phi_{1}$ and $\ldots$ and $\phi_{k}$ then $X$, if $\phi_{1}$ and $\ldots$ and $\phi_{k-1}$ then $X, \ldots$, if $\phi_{2}$ and $\ldots$ and $\phi_{k}$ then $X, \ldots$, if $\phi_{k}$ then $\left.X, X\right\}$.

For instance, the hypothesis space corresponding to $\left\ulcorner\right.$ if $\phi_{1}$ and $\phi_{2}$, then $\psi\{X\}\urcorner$ is $\mathcal{H}=\left\{\right.$ if $\phi_{1}$ and $\phi_{2}$ then $X$, if $\phi_{1}$ then $X$, if $\phi_{2}$ then $\left.X, X\right\}$.

\subsection{Deriving Search Spaces: Local Satisfaction and the Syntax of CCPs}

I arrived at the above generalizations through use of the HWAMT and intuitions about the information conveyed by the relevant sentences. However, I haven't as yet offered a predictive theory. What I have said so far will not be able to tell us, for any given sentence, what its set of alternatives for accommodation should be. This section is devoted to showing that the instructions encoded in CCPs are exactly what is needed to put a predictive theory in place.

There are two important technical notions we need, the notion of a local context and the notion of local satisfaction. Suppose that we wish to execute the CCP of a complex sentence $S$ on a context $c$. Suppose that $\psi$ occurs in an embedded position somewhere in $S$. Since complex sentences come with complex CCPs, we

\footnotetext{
${ }^{16}$ Irene Heim (p.c.) raised the question of how we know that this is indeed the accommodation and not, say, the minimal repair itself. My response is: If we accept this sentence at time $t$, and at some later time $t^{\prime}$ come to learn that John doesn't have a wetsuit, what other information, if any, do we give up? We conclude that John is not a scuba diver, not that John either is not a scuba diver or doesn't want to impress his girlfriend.
} 
will find that $[\psi]$ will be executed on a context $c *$ which is derived from $c$ by application of $\left[\phi_{i}\right]$ for zero or more $\phi_{i}$. Thus, the context on which $[\psi]$ will be executed will be a (not-necessarily proper) subset of $c$.

Definition 1 (Local Contexts) Let $S$ be a sentence uttered in context c, and let $\psi$ be a sentence embedded in $S$. The local context for $\psi$ embedded in $S$ uttered in context $c, \mathcal{L}(\psi, S, c)$, is that subset of $c, c *$, on which $[\psi]$ will be executed, as defined by the CCP of $S$.

For instance, consider the case of atomic, negated, conjunctive, and conditional sentences $S$ uttered in context c:

$c[\psi\{X\}] ; \mathcal{L}(\psi\{X\}, S, c)=c$

$c[$ not $\psi\{X\}]=c-c[\psi\{X\}] ; \mathcal{L}(\psi\{X\}, S, c)=c$

(21) $c[\phi$ and $\psi\{X\}]=c[\phi][\psi\{X\}] ; \mathcal{L}(\psi\{X\}, S, c)=c^{\prime}$, where $c^{\prime}$ is arrived at by executing $[\phi]$ on $c$

(22) $c[$ if $\phi$, then $\psi\{X\}]=c-(c[\phi]-c[\phi][\psi\{X\}]) ; \mathcal{L}(\psi\{X\}, S, c)=c^{\prime}$, where $c^{\prime}$ is arrived at by executing $[\phi]$ on $c$

(23) $c\left[\right.$ if $\phi_{1}$ and $\phi_{2}$, then $\left.\psi\{X\}\right]=c-\left(c\left[\phi_{1}\right]\left[\phi_{2}\right]-c\left[\phi_{1}\right]\left[\phi_{2}\right][\psi\{X\}]\right) ; \mathcal{L}(\psi\{X\}, S, c)=$ $c^{\prime \prime}$, where $c^{\prime \prime}$ is arrived at by first executing $\left[\phi_{1}\right]$ on $c$ and executing $\left[\phi_{2}\right]$ on the result

The second important technical concept is that of local satisfaction. Recall that for atomic $\psi\{X\},[\psi\{X\}]$ is defined on $c$ only if $c$ satisfies $X$, i.e. only if $c$ is a fixed point for $[X]$, i.e. only if $c[X]=c$. The satisfaction theory simply extends this to all embedded sentences:

Definition 2 (Constraint Requiring Local Satisfaction) Let $S$ be a sentence uttered in context $c$, and let $\psi\{X\}$ be embedded in $S$. Then $\mathcal{L}(\psi\{X\}, S, c)$ must satisfy $X$.

Now suppose we want to update a context $c$ with a sentence $S$, where $S$ is one of (19)-(23). What we want to know is, what does $c$ have to satisfy in order for $c[S]$ to be defined (projection)? Second, if accommodation needs to be made, $c[Y][S]$, what values can $Y$ take on? Can this information be predicted from the Constraint Requiring Local Satisfaction?

Given the CCPs of (19)-(23) and the local satisfaction requirement, certain constraints on $c$ do follow. In (19) and (20) $c$ must satisfy $X$ (this can be read off from the above). In (21) and (22), from the fact that $c^{\prime}$ needs to satisfy $X$, it is a simple matter to prove that $c$ needs to satisfy $\ulcorner$ if $\phi$, then $X\urcorner$. In (23) one can show that 
$c$ must satisfy $\left\ulcorner\right.$ if $\phi_{1}$ and $\phi_{2}$, then $\left.X\right\urcorner$. But these are just the minimal requirements imposed on $c$, and so we are just back to the proviso problem. How do these minimal requirements relate to what is actually accommodated? We promised earlier that the space of accommodation possibilities would follow from the instructions encoded in CCPs. Does it?

To make good on our promise, we should be able to take a CCP, and the local satisfaction requirement, and derive the accommodation hypothesis space. Let us pair below the local contexts (that need to satisfy $X$ ) and the hypothesis space for accommodation (given our generalizations in Section 3.2) for each of (19)-(23) to see if this goal can be met:

$$
\begin{aligned}
& \text { Hypothesis Space for (19) }=\{X\} ; \mathcal{L}(\Psi\{X\}, S, c)=c \\
& \text { Hypothesis Space for (20) }=\{X\} ; \mathcal{L}(\Psi\{X\}, S, c)=c
\end{aligned}
$$

Hypothesis Space for $(21)^{17}=\{$ if $\phi$ then $X, X\} ; \mathcal{L}(\psi\{X\}, S, c)=c^{\prime}$

Hypothesis Space for $(22)=\{$ if $\phi$ then $X, X\} ; \mathcal{L}(\psi\{X\}, S, c)=c^{\prime}$

Hypothesis Space for (23) $=\left\{\right.$ if $\phi_{1}$ and $\phi_{2}$ then $X$, if $\phi_{1}$ then $X$, if $\phi_{2}$ then $X$, $X\} ; \mathcal{L}(\psi\{X\}, S, c)=c^{\prime \prime}$

The local context for $\psi\{X\}$ in (19) and (20) is $c$, so we can tell just by inspection that $c$ must satisfy $X$. Our generalization about the hypothesis space here is that it is a singleton set $\{X\}$. The question is, by what principle does the proposition $c$ (the local context for $\psi\{X\}$ correspond to the hypothesis space $\{X\}$ ? In (21) and (22), the local context for $\psi\{X\}$ is $c^{\prime}$, and the hypothesis space is if $\phi$ then $X, X\}$. In (23) the local context for $\psi\{X\}$ is $c^{\prime \prime}$, and the hypothesis space is $\left\{\right.$ if $\phi_{1}$ and $\phi_{2}$ then $X$, if $\phi_{1}$ then $X$, if $\phi_{2}$ then $\left.X, X\right\}$. Again, the question arises: $c^{\prime}$ and $c^{\prime \prime}$ are just propositions, sets of worlds. Why should they correspond in any systematic way to the hypothesis spaces they correspond to? Given $c, c^{\prime}, c^{\prime \prime}$, how can one recover their corresponding hypothesis spaces? There just does not seem to be enough information in propositions to recover the hypothesis spaces I've paired them with above. The satisfaction theory framework, in its bare essentials, does not provide enough resources to help here. However, we will see that allowing the satisfaction theory to exploit an additional informational resource will suffice to derive the generalizations we promised, and will have the additional effect of turning it into a predictive theory of accommodation.

What is this resource? If we make the assumption that we are granted access not just to what the local context is (as a proposition), but to the description of that context as given by the instruction encoded in the CCP, that will be all the information needed to generate our hypothesis spaces. For example, we need access to not only the fact that our local context is $c^{\prime}$, but that we got to $c^{\prime}$ by applying $[\phi]$ to $c, c^{\prime}=c[\phi]$. As such, we need to modify our definition of local contexts: ${ }^{18}$

\footnotetext{
${ }^{17}$ We haven't discussed the case of conjunctions yet, but this hypothesis space will follow from the procedure we develop in this section.

${ }^{18}$ We follow Geurts (1996) and focus our attention on local contexts with the syntax $c\left[\phi_{1}\right] \ldots\left[\phi_{k}\right]$. It should be pointed out, contra Geurts (1996), that this shape of local contexts is not the general
} 
Definition 3 (Local Contexts, Revised Version) The local context for $\psi$ embedded in $S$ uttered in context $c, \mathcal{L}(\psi, S, c)$, is a description $c\left[\phi_{1}\right] \ldots\left[\phi_{k}\right], k \geq 0$, where the CCP of $S$ specifies the instruction $c\left[\phi_{1}\right] \ldots\left[\phi_{k}\right][\psi]$.

Let us return now to our pairing of hypothesis spaces and local contexts:

(29) Hypothesis Space for (19) $=\{X\} ; \mathcal{L}(\psi\{X\}, S, c)=c$

(30) Hypothesis Space for (20) $=\{X\} ; \mathcal{L}(\psi\{X\}, S, c)=c$

(31) Hypothesis Space for (21) $=\{$ if $\phi$ then $X, X\} ; \mathcal{L}(\psi\{X\}, S, c)=c[\phi]$

(32) Hypothesis Space for (22) $=\{$ if $\phi$ then $X, X\} ; \mathcal{L}(\psi\{X\}, S, c)=c[\phi]$

(33) Hypothesis Space for (23) $=\left\{\right.$ if $\phi_{1}$ and $\phi_{2}$ then $X$, if $\phi_{1}$ then $X$, if $\phi_{2}$ then $X$, $X\} ; \mathcal{L}(\psi\{X\}, S, c)=c\left[\phi_{1}\right]\left[\phi_{2}\right]$

\section{Hypothesis Spaces 3 (Procedure for Deriving Hypothesis Spaces from CCPDs)}

Suppose that complex sentence $S$ contains embedded in it sentence $\psi\{X\}$, and that $S$ is uttered in context $c$. Suppose further that $\mathcal{L}(\psi\{X\}, S, c)=c\left[\phi_{1}\right] \ldots\left[\phi_{k}\right]$, where $k \geq 0$. Then the hypothesis space for accommodation (into $c$ ) is: $\left\{\right.$ if $\phi_{1}$ and ... and $\phi_{k}$ then $X$, if $\phi_{1}$ and $\ldots$ and $\phi_{k-1}$ then $X, \ldots$, if $\phi_{2}$ and $\ldots$ and $\phi_{k}$ then $X, \ldots$, if $\phi_{k}$ then $X, X\}$.

When $\mathcal{L}(\psi\{X\}, S, c)=c$, the hypothesis space $\mathcal{H}$ is the singleton set $\{X\}$. When $\mathcal{L}(\psi\{X\}, S, c)=c[\phi]$, such as in sentences of the form $\ulcorner$ if $\phi$ then $\psi\{X\}\urcorner, \mathcal{H}$ $=\{$ if $\phi$ then $X, X\}$. When we have a sentence like (20), of the form $\left\ulcorner\right.$ if $\phi_{1}$ and $\phi_{2}$, then $\left.\psi\{X\}\right\urcorner, \mathcal{L}(\psi\{X\}, S, c)=c\left[\phi_{1}\right]\left[\phi_{2}\right]$, so $\mathcal{H}=\left\{\right.$ if $\phi_{1}$ and $\phi_{2}$ then $X$, if $\phi_{1}$ then $X$, if $\phi_{2}$ then $\left.X, X\right\}$. In general, the size of the hypothesis space corresponding to $\mathcal{L}(\psi\{X\}, S, c)=c\left[\phi_{1}\right] \ldots\left[\phi_{k}\right]$ is $2^{k}$, so that strengthening is only an option when $k>0$.

It is important to note, following Geurts (1996), that "the proviso problem isn't a problem about conditionals - it is also a problem about conditionals." 19 The satisfaction theory predicts that conjunctions $\ulcorner\phi$ and $\psi\{X\}\urcorner$ also generate conditional presuppositions $\ulcorner$ if $\phi$ then $X\urcorner$. However, as pointed out by Geurts, we come away from such sentences inferring something stronger, viz. $X$. Consider the following example:

(34) John works for Morgan Stanley and his limo is parked outside.

We infer $X=$ John has a limo from this sentence. This fact is actually consistent with a conditional presupposition. Assuming that if John works for Morgan Stanley he has a limo is generated as the presupposition of (34), John's having a limo can be inferred by modus ponens from this conditional presupposition together

case. Relevant counter-examples are $\ulcorner($ not $\phi)$ and $\psi\{X\})\urcorner$ (Danny Fox, p.c.) and $\ulcorner$ if $(\alpha$ or $\beta)$, then $\psi\{X\}\urcorner$ (Jeff Pelletier, p.c.). We provide a more general, recursive definition of local contexts in the Appendix. The procedure for generating hypothesis spaces, developed immediately below, also receives a natural extension. I omit details here.

${ }^{19}$ p. 269 , emphasis in original. 
with the first conjunct John works for Morgan Stanley. The inference to John's having a limo can also be explained by generating $X$ itself as (34)'s presupposition. My system predicts exactly these alternative possibilities for accommodation: $\{$ if $\phi$ then $X, X\}$. How then can we show that both members of the hypothesis space are indeed accommodation possibilities, given that we have an independent explanation for the inference to $X$ under each?

When we embed (34) under negation, which I predict to be a hole for presupposition (in that $\ulcorner$ not $\phi\urcorner$ presupposes whatever $\phi$ presupposes), we find that the conditional presupposition if John works for Morgan Stanley he has a limo is indeed available:

(35) It's not the case that (John works for Morgan Stanley and his limo is parked outside).

However, there is no story to be had here tying conditional presuppositions to negated conjunctions, for we accommodate simply that John has a limo in (36):

(36) It's not the case that (it's sunny in Cambridge and John's limo is parked outside).

Thus, like conditionals $\ulcorner$ if $\phi$, then $\psi\{X\}\urcorner$, we see that both members of the predicted search space, $\{$ if $\phi$ then $X, X\}$, are possible repairs.

It is important to note that, although conjunctions and conditionals differ syntactically and semantically in important ways, they share a crucial feature in the syntax of their CCPs, namely, the descriptions of certain local contexts upon which are imposed constraints of local satisfaction. Such an equivalence is hardly obvious from basic assumptions about the syntax or (static) semantics of these constructions, but they follow naturally once CCPDs are taken seriously.

My proposal actually turns this into a more general phenomenon. It will be quite difficult to make generalizations about accommodation based on purely syntactic/semantic properties of sentences unless they bear on the CCPDs of the sentences. Sometimes, as in the case of conditionals and conjunctions, constructions with no obvious syntactic or semantic similiarities, I predict that they will share the same accommodation possibilities. Other times, I predict that truth-conditionally equivalent sentences should converge in their accommodation possibilities. ${ }^{20}$ At other times still, I predict that truth-conditionally equivalent sentences will differ in accommodation possibilities. ${ }^{21}$ Again, the only relevant factor governing the space of accommodation possibilities is the CCPD under consideration.

An implicit assumption expressed in the statement of the proviso problem was that if two sentences $S$ and $S^{\prime}$ generate the same presupposition, they should, ceteris paribus, give rise to the same accommodation possibilities. As I hope to have shown here, ceteris are not always paribus. Even if $S$ and $S^{\prime}$ generate the same

\footnotetext{
${ }^{20}$ As with $\left\ulcorner\right.$ if $\phi_{1}$ and $\phi_{2}$, then $\left.\psi\{X\}\right\urcorner$ and $\left\ulcorner\right.$ if $\phi_{1}$, then if $\phi_{2}$, then $\left.\psi\{X\}\right\urcorner$.

${ }^{21}$ If we assume a symmetric $o r$, so that $c[\phi$ or $\psi]=c[\phi] \cup c[\psi]$, and a material implication analysis of conditionals, then $\ulcorner\phi$ or $\psi\{X\}\urcorner$ is predicted to allow only one accommodation possibility, $\{X\}$, whereas $\ulcorner$ if $\phi$, then $\psi\{X\}\urcorner$, as we've seen, is predicted to allow variabiity, $\{$ if $\phi$ then $X, X\}$.
} 
semantic presupposition, they might nonetheless have CCPDs under which different accommodation possibilities are generated. Thus, although $\ulcorner$ if $\phi$ then $\psi\{X\}\urcorner$ and $\left\ulcorner K_{m}\right.$ (If $\phi$, then $\left.\left.X\right)\right\urcorner$ both generate the same presupposition $\ulcorner$ if $\phi$ then $X\urcorner$, under my proposal they could, in principle, exhibit variability in their accommodation behaviour should their CCPDs dictate that this should be so. In the next section I show that this possibility is actually realized.

\subsection{Embedding Under know}

If we wish to know what accommodation possibilities are generated for some construction, the task for us should be clear. We need to write our lexical entries so as to encode the right semantic content and partiality (where applicable). The syntax of CCPs will then tell us what the set of alternatives for accommodation are.

Assuming we had never heard of the proviso problem, what lexical entry would we write for know sentences? We would need to encode: (i) a definedness condition (if applicable), and (ii) its contribution to content. Let us make this our task. How should we proceed?

One robust feature of the interpretation of such sentences is that from $\left\ulcorner K_{m}(\phi)\right\urcorner$ one tends to infer that $\phi$. This factivity inference seems to arise not through standard entailment, but through presupposition, as standard projection tests show:

(37) If Mary knows that James Baldwin wrote Notes of a Native Son, she probably has two copies at home

Thus our definedness condition will include a statement like the following:

Definition 4 (Definedness of Knowledge-Attributions) $c\left[K_{m}(\phi)\right]$ is defined only if c satisfies $\phi$, i.e. only if $c[\phi]=c$.

Note that from this definedness condition follows another, viz. that $c[\phi]$ must be defined. Thus, if $\phi$ itself is presuppositional, eg. if $\phi$ presupposes $X$, then for $c[\phi]$ to be defined it must be that $c$ satisfies $X$. As a result, $\left[K_{m}(\phi\{X\})\right]$ will be defined on $c$ only if (i) $c$ satisfies $X$, and (ii) $c$ satisfies $\phi$. This is the familiar "hole" property of knowledge attribution sentences. For instance, (38) is predicted to presuppose both that John has a sister, and that John's sister will pick him up from the airport.

(38) Mary knows that John's sister will pick him up from the airport

Before even writing the content of knowledge attribution sentences, we are in position to say why conditional presuppositions generated under know are never strengthened. ${ }^{22}$ Consider a sentence like (3), repeated below as (39), uttered in

\footnotetext{
${ }^{22}$ See the appendix for a full lexical entry, modelled after the semantics of belief attribution developed in Heim (1992). Once our entry is spelled out, the CCP will add a further definedness condition, viz. that $\left[K_{m}(\phi\{X\})\right]$ is defined on context $c$ only if $c$ satisfies $K_{m}(X)$. This would mean that (38), eg. presupposes that Mary knows that John has a sister. Crucially, this definedness condition is not stipulated, but follows from the basic compositional semantics we assign to know. Thus, from two assumptions: (i) the basic definedness condition of factivity, and (ii) the semantic contribution of know, we derive two new definedness conditions.
} 
context $c$ :

(39) Mary knows that if John flies to Toronto, he has a sister

The first step in the update is to check for definedness. This means we check whether $c$ satisfies the complement of know, i.e. we check whether $c$ [if John flies to Toronto, he has a sister] $=c$. Since $\mathrm{k}=0$, i.e. since the local context that needs to satisfy [if John flies to Toronto, he has a sister] is just $c$ itself, and not some more complex context of the form $c\left[\phi_{1}\right] \ldots\left[\phi_{k}\right], k>0$, there is no option for strengthening (cf. Section 4.3). Our procedure generates only the following singleton set as the accommodation hypothesis space: $\{$ if John flies to Toronto, he has a sister\}. This is the reason why there is no strengthening under know.

\section{Brief Comparison with the Binding Theory of Presupposition}

My analysis of presupposition accommodation is, like the binding theory (van der Sandt 1992, Geurts 1999), rather syntactic in nature. I believe there is no escape from this conclusion. Geurts's critique of the satisfaction theory teaches us that we have to take alternatives for accommodation seriously. Moreover, the binding theory not only takes formal alternatives seriously, but it also proposes an explicit algorithm which, given any sentence, produces a finite set of accommodation possibilities. Now that the satisfaction theory has a proposal in place for predicting the space of accommodation possibilities, it makes it possible to compare the predictions of the two theories. ${ }^{23}$ Here, we can only point to some of the ways in which the theories differ in predictions.

First, consider again conditionals of the form $\ulcorner$ if $\phi$, then $\psi\{X\}\urcorner$, such as (1) and (2). We repeat these below as (40) and (41):

(40) If John flies to Toronto, his sister will pick him up from the airport

(41) If John is a scuba diver, he'll bring his wetsuit

I predict that once accommodation possibilities are incorporated such sentences can only have two interpretations each, ${ }^{24}$ which can be paraphrased as below:

(42) John has a sister and if he flies to Toronto, his sister will pick him up from the airport

(43) If John flies to Toronto, then he has a sister and she will pick him up from the airport

\footnotetext{
${ }^{23}$ Kai von Fintel (p.c.) has raised the question of the extent to which my proposal counts as a version of the satisfaction theory, given that it uses an additional level of representation (CCPDs). Although I deviate from the letter of the satisfaction theory, I leave it to others to judge the extent to which this proposal remains in the spirit of the theory. For what it's worth, I believe my proposal constitutes a somewhat conservative extension of the theory, once we take seriously the notion of CCPs as specifying "instructions" for context change.

${ }^{24}$ At the level of accommodation + content.
} 
(44) John has a wetsuit and if he's a scuba diver, he'll bring his wetsuit

(45) If John is a scuba diver, he has a wetsuit and will bring it

Of course, (42) is the preferred interpretation of (40) (which corresponds to accommodation of $X$ ), and (45) is the preferred interpretation of (41) (which corresponds to accommodation of $\ulcorner$ if $\phi$ then $X\urcorner$ ). In general, we predict that $\ulcorner$ if $\phi$, then $\psi\{X\}\urcorner$ can only be interpreted in one of two ways:

$\ulcorner X$ and (if $\phi$, then $\psi\{X\})\urcorner$

$\ulcorner$ if $\phi$, then $(X$ and $\psi\{X\})\urcorner$

The binding theory predicts these two readings, plus a third, the so-called "intermediate accommodation" reading:

$\ulcorner$ if $X$ and $\phi$, then $\psi\{X\}\urcorner$

In (40) and (41), this would correspond to the following paraphrases:

(49) If John has a sister and he flies to Toronto, his sister will pick him up from the airport

(50) If John has a wetsuit and is a scuba diver, he'll bring his wetsuit

As far as I can tell, conditionals $\ulcorner$ if $\phi$, then $\psi\{X\}\urcorner$ don't generate such a reading. ${ }^{25}$

A second issue arises with the binding theory's binding/accommodation procedure. For example, if in the antecedent of a conditional a discourse referent is introduced by an indefinite, and the consequent contains a pronoun/definite description, then both the pronoun and the definite description should prefer to be read as "bound" by the discourse referent. At least, nothing should rule out such a reading. However, the following example shows this to be a problematic prediction:

Context: John is married to three women, though we are fully ignorant about their nationalities.

(51) If John is married to an American, she is a great cook

(52) \#If John is married to an American, his wife is a great cook

In (52), the binding/accommodation procedure should allow his wife to ignore the other wives and pick up the American introduced in the antecedent, just as it allows she to do so in (51). The satisfaction theory on the other hand is not tied to such a prediction. In fact, it makes it rather explicit that the analogy between pronouns and definites should not be pushed too far (cf. Heim (1982)).

Much more deserves to be said here. I haven't shown how my definition of local contexts generalizes beyond connectives (eg. to quantifiers and attitude

\footnotetext{
${ }^{25}$ For more debate on the issue of intermediate accommodation see Geurts and van der Sandt (1999) for arguments supporting intermediate accommodation, and Beaver (2001, 2004), von Fintel (2004a, 2006) for arguments against. See especially Beaver (2001) for extended discussion.
} 
predicates). I also haven't said how the alternatives I offer here might be usable by other theories that predict conditional presuppositions. I also need to respond to the charge that CCPDs are, to some degree, stipulated (Soames 1989, Mats Rooth in a personal letter to Irene Heim (Heim 1990), Schlenker 2007). Regarding the latter, one may view this paper as accepting the stipulated entries, but providing an independent argument for their correctness. To the extent that the argument is sound, I hope to movitate the search for a justification for the entries.

\section{Appendix}

A 1 (Recursive Definition of Context Descriptions) Let $W$ be the set of all worlds, $\phi$ a sentence. Any $c \subset W$ is a context description. Then, for any context descriptions $\sigma, \delta:(i) \sigma[\phi]$ is context description, (ii) $\sigma-\delta$ is a context description, (iii) $\sigma \cup \delta$ is a context description, (iv) nothing else is a context description.

A 2 (The CCP of Knowledge Attribution Sentences) For any world $w$, where $R_{m}$ is the accessibility relation for Mary, let Dox $x_{m}(w)=\left\{w^{\prime} \in W: w R_{m} w^{\prime}\right\}$. Then $c\left[K_{m}(\phi)\right]=\left\{w \in c: \operatorname{Dox}_{m}(w)[\phi]=\operatorname{Dox}_{m}(w)\right\}$, Since the entry is for know, the relation will be reflexive, symmetric, and transitive, validating an S5 modal logic. It follows from this CCP, plus what we said in the text, that $c\left[K_{m}(\phi\{X\})\right]$ is defined only if: (i) $c[X]=c$, (ii) $c[\phi]=c$, (iii) $c\left[K_{m}(X)\right]=c$.

\section{References}

Beaver, David: 2001, Presupposition and Assertion in Dynamic Semantics. CSLI Publications, Stanford, CA.

Beaver, David: 2004, 'Accommodating Topics', in H. Kamp and B. H. Partee (eds.), Context-Dependence in the Analysis of Linguistic Meaning. Elsevier, Amsterdam.

Beaver, David: 2006, 'The Proviso Problem: Comments on von Fintel'. Handout from Ohio State University Accommodation Workshop, Columbus, Ohio.

Beaver, David and Henk Zeevat: To appear, 'Accommodation', in G. Ramchand and C. Reiss (eds.), Oxford Handbook of Linguistic Interfaces. Oxford University Press, Oxford.

von Fintel, Kai: 2004a, 'Comments on Beaver: Presupposition Accommodation and Quantifier Domains', in H. Kamp and B. H. Partee (eds.), ContextDependence in the Analysis of Linguistic Meaning. Elsevier, Amsterdam.

von Fintel, Kai: 2004b, 'Notes on Presupposition'. Lecture Notes, 24.954: Pragmatics in Linguistic Theory, MIT.

von Fintel, Kai: 2004c, 'Would You Believe It? The King of France is Back! Presuppositions and Truth-Value Intuitions', in M. Reimer and A. Bezuidenhout (eds.), Descriptions and Beyond. Oxford University Press, Oxford. 
von Fintel, Kai: 2006, 'What is Presupposition Accommodation, Again?'. Manuscript, MIT.

Gazdar, Gerald: 1979, Pragmatics. Academic Press, New York, NY.

Geurts, Bart: 1996, 'Local Satisfaction Guaranteed: A Presupposition Theory and its Problems', Linguistics and Philosophy 19, 259-294.

Geurts, Bart: 1999, Presuppositions and Pronouns. Elsevier, Amsterdam.

Geurts, Bart and Rob van der Sandt: 1999, 'Domain Restriction', in P. Bosch and R. van der Sandt (eds.), Focus: Linguistic, Cognitive, and Computational Perspectives. Cambridge University Press, Cambridge.

Heim, Irene: 1982, 'On the Semantics of Definite and Indefinite Noun Phrases'. Doctoral Dissertation, University of Massachusetts at Amherst.

Heim, Irene: 1983, 'On the Projection Problem for Presuppositions', in WCCFL 2, 114-125.

Heim, Irene: 1990, 'Presupposition Projection', in Reader for the Nijmegen Workshop on Presupposition, Lexical Meaning and Discourse Processes. University of Nijmegen, Nijmegen.

Heim, Irene: 1992, 'Presupposition Projection and the Semantics of Attitude Verbs', Journal of Semantics 9, 183-221.

Heim, Irene: 2006, 'A Note on the Proviso Problem'. Handout from Milan Meeting, Gargnano.

Karttunen, Lauri: 1974, 'Presupposition and Linguistic Context', Theoretical Linguistics 1, 181-193.

Karttunen, Lauri and Stanley Peters: 1979, 'Conventional Implicatures in Montague Grammar', in C.-K. Oh and D. Dineen (eds.), Syntax and Semantics 11: Presupposition, 1-56. Academic Press, New York, NY.

Lewis, David: 1979, 'Scorekeeping in a Language Game', Journal of Philosophical Logic 1, 339-359.

Pérez Carballo, Alejandro: 2007, 'A First Shot at the Proviso Problem'. Manuscript, MIT.

van Rooij, Robert: 2007, 'Strengthening Conditional Presuppositions', Journal of Semantics 24, 289-304.

van der Sandt, Rob: 1992, 'Presupposition Projection as Anaphora Resolution', Journal of Semantics 9, 333-377.

Schlenker, Philippe: 2007, 'Anti-Dynamics: Presupposition Projection Without Dynamic Semantics', Journal of Logic, Language, and Information 16, 325-356.

Soames, Scott: 1989, 'Presupposition', in D. Gabbay and F. Guenther (eds.), Handbook of Philosophical Logic, vol. IV. Reidel, Dordrecht.

Stalnaker, Robert: 1998, 'On the Representation of Context', Journal of Logic, Language, and Information 7, 3-19.

Thomason, Richmond H., Matthew Stone, and David DeVault: 2006, 'Enlightened Update: A Computational Architecture for Presupposition and other Pragmatic Phenomena'. Manuscript, University of Michigan and Rutgers University. 